\section{Best of International Stereotactic Radiosurgery Society Congress 2013: stereotactic body radiation therapy. Part l: spinal tumors}

Simon S Lo*1, Eric L Chang ${ }^{2}$, Samuel Ryu ${ }^{3}$, Hans Chung $^{4}$, Ben J Slotman ${ }^{5}$, Bin S Teh ${ }^{6} \&$ Arjun Sahgal $\left.\right|^{4,7}$ 'Department of Radiation Oncology, University Hospitals Seidman Cancer Center, Case Comprehensive
Cancer Center, Cleveland, OH, USA

2Department of Radiation Oncology, Norris Cancer Center \& Keck School of Medicine, University of Southern California, Los Angeles, CA, USA

3.Department of Radiation Oncology, Henry Ford Hospital, Detroit, MI, USA

${ }^{4}$ Department of Radiation Oncology, Odette Cancer Centre, University of Toronto, Toronto, ON, Canada

5Department of Radiation Oncology, VU University Medical Center, Amsterdam, The Netherlands

bDepartment of Radiation Oncology, The Methodist Hospital \& Weill Cornell Medical College, Houston,

TX, USA

'Department of Radiation Oncology, Princess Margaret Cancer Centre, University of Toronto, Toronto, ON,

Canada

*Author for correspondence: Tel. : +1 2168443103 = Fax: +1 2168442005 = simon.lo@UHhospitals.org

\section{1th International Stereotactic Radiosurgery Society Congress}

\section{6-20 June 2013, Toronto, ON, Canada}

The 11th biennial International Stereotactic Radiosurgery Society Congress represented another historical gathering of professionals in the field of stereotactic radiosurgery. This congress was held on 16-20 June 2013 in Toronto (ON, Canada), and the chairman was Arjun Sahgal, the co-chair was Michael Schwartz and president of the society was Jean Regis. The congress attracted 550 attendants from all over the world and over 300 abstracts were presented. Among the abstracts presented, 62 (36 oral) were pertaining to stereotactic body radiation therapy (SBRT). Exciting new findings were presented by colleagues from North America, Europe and Asia. This short conference scene (part I) provides a summary of the best abstracts on SBRT for spinal tumors presented in the congress. $A$ separate conference scene on SBRT for nonspinal tumors (part II) also appears in this issue of Future Oncology.

\section{Spinal tumors}

The Radiation Therapy Oncology Group is conducting a Phase II/III trial of stereotactic body radiation therapy (SBRT) for spinal metastasis (RTOG 0631) and preliminary results demonstrating the feasibility and safety of the Phase II component were presented in the plenary session of the International Stereotactic Radiosurgery Society congress by Ryu [1]. Forty-one patients each with one to three spinal metastases were enrolled in the study and each lesion was treated to a dose of $16 \mathrm{~Gy}$ in one fraction. Among them, 36 (30 with one lesion and six with two lesions) had on-study information. All the patients were successfully treated with SBRT according to protocol guidelines, with spinal cord constraints met in all patients. All the patients had optimal (91\%) or acceptable image-guided radiotherapy compliance. Grade 1-2 and grade 3-5 SBRTrelated adverse events were observed in seven and zero patients, respectively. Grade 3-4 nonSBRT-related adverse events were observed in four patients [1]. Clinical outcomes with respect to local control (LC) and pain control will follow as data mature, and the Phase III portion of the trial comparing conventional radiotherapy to a dose of $8 \mathrm{~Gy}$ in one fraction and SBRT to a dose of 16-18 Gy is ongoing.

Colleagues from University of Toronto (ON, Canada) reported several important studies in spinal SBRT. In one study, Sahgal's group assessed spinal cord motion in spinal SBRT with the use of dynamic axial and sagittal MRI in 33 patients [2]. They found that the median physiologic oscillatory spinal cord motion in the anteroposterior, lateral and superoinferior directions were $0.17,0.20$ and $0.24 \mathrm{~mm}$, respectively. The corresponding maximum values were 0.92 , 0.93 and $0.83 \mathrm{~mm}$, respectively. Corresponding bulk displacements from gross patient motion were $0.44 \mathrm{~mm}$ (median) $/ 1.77 \mathrm{~mm}$ (maximum), $0.52 \mathrm{~mm}$ (median) $/ 2.87 \mathrm{~mm}$ (maximum) and $0.59 \mathrm{~mm}$ (median)/3.90 mm (maximum), respectively [2]. Bulk displacements were more
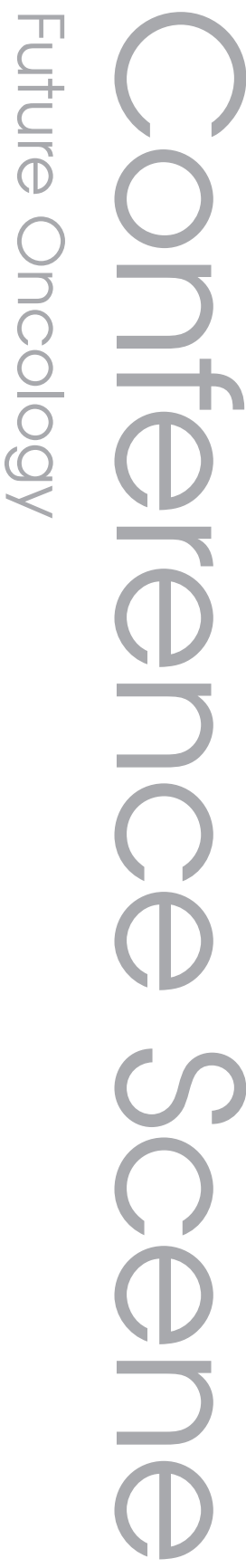

\section{Keywords}

- International Stereotactic Radiosurgery Society - spinal tumors $=$ stereotactic body radiation therapy

Future 
than $1.5 \mathrm{~mm}$ in $5.6,11.1$ and $16.7 \%$ of vertebral levels in the anteroposterior, lateral and superoinferior directions, respectively [2]. The findings of this study underscore the importance of robust immobilization and the use of planning organ at risk volume for spinal cord during treatment planning of spinal SBRT. In another study, Becker N et al. from the University of Toronto found that with the addition of flattening-filter-free beams for volumetric modulated arc therapy, the treatment delivery times could be reduced by 57 and $75 \%$ for 6 and $10 \mathrm{MV}$, respectively [3]. Hyde et al., also from the University of Toronto, examined the impact of treating multiple consecutive vertebrae as a single volume with spinal SBRT on positional accuracy based on 415 verification cone-beam computed tomography images. A total of 25,16 , 20 and six treatments were given for single thoracic vertebrae, multiple thoracic vertebrae, single lumbar vertebrae and multiple lumbar vertebrae, respectively [4]. The absolute intrafraction translational motion averaged over all directions for single thoracic vertebrae, multiple thoracic vertebrae, single lumbar vertebrae, and multiple lumbar vertebrae were $0.54,0.54,0.36$ and $0.47 \mathrm{~mm}$, respectively. The corresponding absolute intrafraction rotational motion averaged over all directions were $0.31^{\circ}, 0.26^{\circ}, 0.23^{\circ}$ and $0.27^{\circ}$, respectively [4]. The percentage of cases that were not covered by the 1.5 -mm planning target volume margin were 3.8 , 4.0, 1.0 and $0.85 \%$, respectively. A statistically significant difference between single and multiple spinal segments was only observed for lumbar and not thoracic vertebrae, although there were more treatments that went beyond tolerance for thoracic vertebrae [4]. This study underscores the importance of planning a target volume margin expansion for spinal SBRT and very close monitoring of intrafraction motion, especially when multiple lumbar vertebrae are included in a single volume.

The outcomes for patients with renal cell carcinoma (RCC) spinal metastases and postoperative spinal metastasis patients treated with SBRT at the University of Toronto were also presented [5,6]. In the first study, outcomes from 37 patients with 71 RCC spinal metastases treated with SBRT to a dose of 18-30 Gy in one to five fractions (median: $24 \mathrm{~Gy}$ in two fractions) were presented. The 1-year overall survival (OS) and LC rates were 64 and $83 \%$, respectively [5]. The most common site of failure was epidural space, with two out of three of the lesions that failed occurring there. Oligometastatic status was the only factor predicting OS [5]. In the second study, 80 patients with spinal metastases were treated with postoperative SBRT. The 1-year LC and OS rates were $84 \%$ and $63 \%$, respectively [6]. Failure in the epidural space occurred in $71 \%$ of the lesions that failed. Treatment with one or two fractions and postoperative grade 0 or 1 epidural disease predicted LC [6]. The findings of these studies were in keeping with the observations from other series where one of the most common places for failure was the epidural space, and this was most likely due to underdosing of the epidural disease caused by aggressive attempts to spare the spinal cord or poor tumor biology. The postoperative series was of high importance as it showed for the first time the value of aggressive debulking of epidural disease to maximize LC outcomes following SBRT. [7]. Since both studies had median follow-up intervals of $\leq 12$ months, a much longer follow-up is required to determine the long-term LC and confirm results.

Pain flare and vertebral compression fractures (VCFs) are commonly encountered complications of spinal SBRT and colleagues from the University of Toronto who have a large body of experience with this technique presented three papers pertaining to these issues. In the first paper, Thibault et al. presented data on VCFs after SBRT for spinal metastases from RCC $[8]$. The observed incidence was $16 \%$, which is similar to a prior study that included all histologies from the same group [9]. The only predictive factor was baseline VCFs [8]. It does not appear that spinal metastases from RCC are significantly different from other histologies in terms of risk of VCF after SBRT. In the second paper, Chiang et al. prospectively evaluated pain flare associated with SBRT for spinal metastasis, and the incidence was striking at $68.3 \%$ [10]. This paper has since been published in International Journal of Radiation Oncology, Biology and Physics after abstract submission [11]. In the third study [12], which has since been published in Journal of Neurosurgery Spine after abstract submission [13], the clinical, radiologic and pathologic findings of two patients with radiographic evidence of late VCF after SBRT for spinal metastasis were presented. Radiation necrosis and radiation fibrosis were observed in those two patients after $20 \mathrm{~Gy}$ in one fraction and $24 \mathrm{~Gy}$ in two fractions, respectively [13]. Biopsy is recommended by the group if it is uncertain whether the marrow signal changes represent radiation-induced effect or tumor progression. Colleagues from University of Pittsburgh (PA, USA) also presented the findings of histologic examination of spinal metastases after SBRT, although in a much larger series. Among the 222 patients treated with single fraction SBRT to a dose of 14-20 Gy, 15 required subsequent surgery. Ten out of the 15 had surgery for suspected 
progressive disease causing compression of spinal cord or cauda equina and five out of the 15 for symptomatic VCF and/or mechanical instability [14]. Thirteen cases showed varying degrees of inflammation. Among the ten cases with suspected progression, under the light microscope, nine showed a tumor within the specimen, two showed ectatic vessels, nine had fibrotic marrow and nine had necrosis [14]. The two latter studies help spinal SBRT practitioners to better understand the pathophysiologic changes in spinal metastases treated with ablative radiotherapy.

Chang et al. from the Korea Institute of Radiological and Medical Science (Seoul, South Korea), presented their outcomes on SBRT for spinal metastases from hepatocellular carcinoma. Twenty seven patients with 39 spinal metastases were treated with CyberKnife ${ }^{\circledR}$ (Accuray, CA, USA) SBRT to a single equivalent dose of $20 \mathrm{~Gy}$ (range: 12-28.5 Gy). The local recurrence rate was $23.1 \%$ [15]. Pain control was achieved in $84.6 \%$ of the lesions and $21.2 \%$ had recurrent pain. The median OS and progression-free survival (PFS) rates were 8 ( 19 for age $\leq 52$ years and 7 for $>52$ years) and 7 months, respectively [15]. Patients who had prior radiotherapy had poorer PFS. No neurologic complications were observed. Given the short mean follow-up of 11.1 months, continued follow-up is necessary to determine long-term PFS. This is the largest series on hepatocellular carcinoma spine SBRT known to date.

Sohn et al. from Inje University Ilsan Paik Hospital (Goyang, South Korea) presented their experience with SBRT for benign intradural extramedullary spinal tumors. Sixty-two patients with neurogenic tumors ( 41 schwannomas and 11 neurofibromas) and meningiomas (ten) were treated with SBRT using mostly a single-fraction regimen, delivering 13 and 15 Gy to neurogenic tumors and meningiomas, respectively [16]. Eight patients with giant presacral and intraosseous schwannomas, or en-plaque type or atypical meningiomas were treated with 2-5 Gy. Two patients with neurofibromas developed malignant transformation of their SBRT-treated tumors, and one patient with atypical meningioma developed neurologic toxicity [16]. Four patients required repeat SBRT for either newly developed neurogenic tumors or recurrent meningiomas. Pain relief was achieved in the three patients with giant intraosseous schwannomas. Marchetti et al. from Fondazione IRCCS Istituto Neurologico C Besta (Milan, Italy), presented their results on the use of SBRT for intradural benign spinal tumors in 20 patients with 24 tumors (14 meningiomas, nine schwannomas and one neurofibroma). Eleven lesions were treated with single fraction SBRT to a dose of 10-15 Gy and the rest were treated with multisession SBRT to a dose of 16.8-30 Gy in 4-6 Gy fractions [17]. At a median follow-up of 43 months, the freedomfrom-progression rate was $100 \%$. Neurologic status was either preserved or improved and there were no permanent treatment-induced sequelae. Significant and durable pain relief was achieved in all lesions with pain [17]. While the results of the above two studies were promising, given the fact that benign spinal tumors typically grow slowly, a much longer follow-up is required to better define the role of SBRT in this group of tumors. The malignant transformation of neurofibroma induced by radiotherapy is a real risk and this was once again demonstrated in the study by Sohn et al. study [16].

\section{Conclusion}

The spine SBRT sessions at the International Stereotactic Radiosurgery Society were a major success with high scientific quality abstracts presented.

\section{Financial \& competing interests disclosure \\ $S$ Ryu is President-Elect of International Stereotactic Radiosurgery Society. BJ Slotman has a research agreement and recieves travel support and speaker honorarium with Varian Medical Systems and BrainLAB AG. A Sahgal is the Chairman of the 2013 International Stereotactic Radiosurgery Society Congress. The authors have no other relevant affilia- tions or financial involvement with any organization or entity with a financial interest in or financial con- flict with the subject matter or materials discussed in the manuscript apart from those disclosed. \\ No writing assistance was utilized in the production of this manuscript.}

\section{References}

1. Ryu S, James JL, Gerszten PC et al. RTOG 0631 Phase II/III study of image-guided stereotactic radiosurgery/SBRT for localized (1-3) spine metastases: Phase II results. Int. J. Radiat. Oncol. Biol. Phys. 81(2) S131-S132 (2011).
2. Tseng C, Sussman M, Simeonov A, Letourneau D, Yu E, Sahgal A. Assessment of spinal cord motion in spine stereotactic body radiotherapy (SBRT). J. Radiosurg. SBRT 2(Suppl. 1), Abstract 202 (2013).

3. Becker N, Sahgal A, Sharpe M et al. Implementation of volumetric modulated arc therapy with flattening filter-free beams for steriotactic body radiotherapy (SBRT) of spinal/paraspinal tumours. J. Radiosurg. SBRT 2(Suppl. 1), Abstract 225 (2013).

4. Hyde D, Sangha A, Korol R, Lochray F, Sahgal A. The impact of treating multiple consecutive vertebrae as a single volume with 
spine stereotactic body radiotherapy (SBRT) on positional accuracy. J. Radiosurg. SBRT 2(Suppl. 1), Abstract 200 (2013).

5. Thiabault I, Al-Omair AS, Masucci GL et al. Spine stereotactic radiotherapy for metastatic renal cell cancer: local control and analysis of predictive and prognostic factors. J. Radiosurg. SBRT 2(Suppl. 1), Abstract 170 (2013).

6. Al-Omair AS, Masucci GL, Masson-Cote L et al. Post-operative stereotactic body radiotherapy (SBRT) for patients with spinal metastasis: predictive and prognostic factors analysis. J. Radiosurg. SBRT2(Suppl. 1), Abstract 298 (2013).

7. Sahgal A, Bilsky M, Chang EL et al. Stereotactic body radiotherapy for spinal metastases: current status, with a focus on its application in the postoperative patient. J. Neurosurg. Spine 14(2), 151-166 (2011).

8. Thiabault I, Al-Omair AS, Masucci GL et al. Vertebral compression fracture after stereotactic radiotherapy for renal cell cancer metastases. J. Radiosurg. SBRT 2(Suppl. 1), Abstract 174 (2013).
9. Lo SS, Sahgal A, Chang EL et al. Serious complications associated with stereotactic ablative radiotherapy and strategies to mitigate the risk. Clin. Oncol. 25(6), 378-387 (2013).

10. Chiang A, Zeng L, Koo K et al. Incidence of pain flare following stereotactic body radiotherapy for spinal metastases. J. Radiosurg. SBRT 2(Suppl. 1), Abstract 275 (2013).

11. Chiang A, Zeng L, Zhang L et al. Pain flare is a common adverse event in steroid-naive patients after spine stereotactic body radiation therapy: a prospective clinical trial. Int. J. Radiat. Oncol. Biol. Phys. 86(4), 638-642 (2013).

12. Al-Omair AS, Smith R, KIehl T et al. Radiation-induced vertebral compression fracture following spine stereotactic radiosurgery: clinical-pathological correlation. J. Neurosurg. Spine 18(5), 430-435 (2013).

13. Al-Omair AS, Smith R, KIehl T et al. Radiation-induced vertebral compression fracture following spine stereotactic radiosurgery: clinical-pathological correlation. J. Radiosurg. SBRT2(Suppl. 1), Abstract 32 (2013).

14. Zwagerman NT, Hamilton R, Monaco EA, Chen S, Flickinger J, Gerszten PC. Histopathological examination of spine metastases after radiosurgery. J. Radiosurg. SBRT2(Suppl. 1), Abstract 179 (2013).

15. Chang U, Lee DH, Rhee CH, Kim MS. Clinical result of stereotactic radiosurgery for spinal metastasis from hepatocellular carcinoma. J. Radiosurg. SBRT 2(Suppl. 1), Abstract 208 (2013).

16. Sohn M, Lee DJ, Hwang YJ. Clinical analysis of spinal stereotactic radiosurgery for benign intradural extramedullary tumors. J. Radiosurg. SBRT 2(Suppl. 1), Abstract 239 (2013).

17. Marchetti MC, Milanesi I, De Martin E, Broggi G, Saini M, Fariselli L. Intradural extramedullary benign spinal lesion radiosurgery. Medium-term results. J. Radiosurg. SBRT2(Suppl. 1), Abstract 45 (2013). 